\title{
Management of thoracic spinal cord injury in a professional American football athlete: illustrative case
}

\author{
Joseph C. Maroon, MD, ${ }^{1}$ Andrew Faramand, MD, MSc, ${ }^{1}$ Nitin Agarwal, MD, ${ }^{1}$ Amanda L. Harrington, MD, ${ }^{2}$ Vikas Agarwal, MD, ${ }^{3}$ \\ John Norwig, MEd, ATC, ${ }^{4}$ and David O. Okonkwo, MD, PhD ${ }^{1}$

\begin{abstract}
Departments of ${ }^{1}$ Neurological Surgery, ${ }^{2}$ Physical Medicine and Rehabilitation, and ${ }^{3}$ Radiology, University of Pittsburgh Medical Center, Pittsburgh, Pennsylvania; and
\end{abstract} \\ ${ }^{4}$ Pittsburgh Steelers, Pittsburgh, Pennsylvania
}

\begin{abstract}
BACKGROUND A case of catastrophic thoracic spinal cord injury (SCl) sustained by a professional American football player with severe scoliosis is presented.

OBSERVATIONS A 25-year-old professional football player sustained an axial loading injury while tackling. Examination revealed a T8 American Spinal Injury Association Impairment Scale grade A complete SCI. Methylprednisolone and hypothermia protocols were initiated. Computed tomography scan of the thoracic spine demonstrated T8 and T9 facet fractures on the left at the apex of a $42^{\circ}$ idiopathic scoliotic deformity. Magnetic resonance imaging (MRI) demonstrated T2 spinal cord hyperintensity at T9. He regained trace movement of his right lower extremity over 12 hours, which was absent on posttrauma day 2. Repeat MRI revealed interval cord compression and worsening of T2 signal change at T7-T8 secondary to hematoma. Urgent decompression and fusion from T8 to T10 were performed. Additional treatment included high-dose omega-3 fatty acids and hyperbaric oxygen therapy. A 2-month inpatient spinal cord rehabilitation program was followed by prolonged outpatient physical therapy. He currently can run and jump with minimal residual distal left lower limb spasticity.
\end{abstract}

LESSONS This is the first known football-related thoracic SCl with idiopathic scoliosis. Aggressive medical and surgical intervention with intensive rehabilitation formed the treatment protocol, with a favorable outcome achieved.

https://thejns.org/doi/abs/10.3171/CASE21206

KEYWORDS spinal cord injury; sports medicine; football; steroids; hypothermia; hyperbaric oxygen therapy; rehabilitation

Since 2010 , football has accounted for only $0.24 \%$ of sport-related spinal cord injuries (SCls) seen at Model Systems Centers nationally. ${ }^{1} \mathrm{~A}$ study of National Football League (NFL) spinal cord and axial skeletonrelated injuries revealed that between 2000 and $2010,44 \%$ of spinal and axial skeleton-related injuries affected the cervical spine. ${ }^{2-4}$ Injuries to the thoracic spine are rare in NFL players, constituting approximately $3.9 \%$ of total spine injuries, with SCls only accounting for $0.6 \%$ of total injuries. ${ }^{2}$ In this report, we present the case of a catastrophic thoracic SCl sustained by a professional American football player. We discuss the multimodality management used and the neurological outcome.

\section{Illustrative Case}

A 25-year-old male NFL professional player sustained an axial loading injury while tackling. On-field examination revealed complete bilateral loss of sensory and motor function below T8. He was transported on a spine board by ambulance to the local trauma center.

Within 30 minutes of injury, a repeat examination demonstrated no perianal sensation and flaccid paralysis of his legs with intact bulbocavernosus reflex. His initial International Standards for Neurological Classification of Spinal Cord Injury (ISNCSCI) examination in the trauma bay was T8 American Spinal Injury Association Impairment

ABBREVIATIONS AIS = American Spinal Injury Association Impairment Scale; $A T A=$ atmopsheres absolute; DHA = docosahexaenoic acid; EPA = eicosapentaenoic acid; HBOT = hyperbaric oxygen therapy; IPR = inpatient rehabilitation; ISNCSCI = International Standards for Neurological Classification of Spinal Cord Injury; MEP = motor evoked potential; MRI = magnetic resonance imaging; NFL = National Football League; $\mathrm{PT}=$ physical therapy; $\mathrm{SCl}=$ spinal cord injury; SSEP = somatosensory evoked potential.

INCLUDE WHEN CITING Published August 9, 2021; DOI: 10.3171/CASE21206.

SUBMITTED May 18, 2021. ACCEPTED June 9, 2021.

(C) 2021 The authors, CC BY-NC-ND 4.0 (http://creativecommons.org/licenses/by-nc-nd/4.0/). 

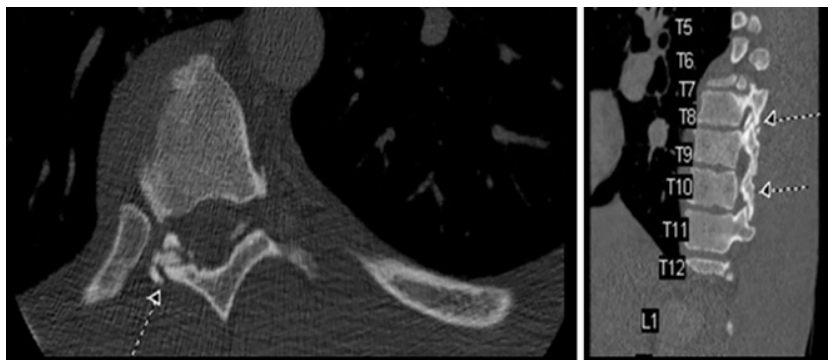

FIG. 1. Initial computed tomography scan obtained at presentation. Axial (left) and sagittal reconstruction (right) views demonstrate T8 and $\mathrm{T} 9$ facet fractures on the right.

Scale (AIS) grade A, indicating a complete lesion. During the trauma resuscitation phase, the patient received $2 \mathrm{~L}$ of chilled saline. The Bracken methylprednisolone protocol ${ }^{5}$ was initiated, and he was placed on a norepinephrine infusion to maintain a mean arterial pressure $>85$ $\mathrm{mm} \mathrm{Hg}$. A computed tomography scan of the thoracic spine demonstrated T8 and T9 facet fractures on the right occurring at the apex of a $42^{\circ}$ idiopathic dextroscoliosis (Fig. 1) without canal compromise. Magnetic resonance imaging (MRI) demonstrated T2 hyperintensity in the spinal cord centered at T8 without neural element compression (Fig. 2 left).

Upon admission to the neurological intensive care unit, hypothermia to $35^{\circ} \mathrm{C}$ was instituted with a surface-cooling device. ${ }^{6}$ Within 3 hours of injury, the patient regained trace motor function in his right great toe; over 12 hours, he regained flicker muscle firing in his right quadriceps and right extensor hallucis longus as well as vague proprioceptive sense. The rectal tone was weak but present. His ISNCSCI examination was reclassified after resuscitation to a T8 AIS C.

On posttrauma day 1 , neurological examination revealed $2 / 5$ strength with right hip, knee, plantar flexion, and right extensor hallucis longus on the British Medical Research Council motor grading scale. He also regained proprioception in the right great toe. He remained plegic (0/5 strength) in his left leg with no pinprick sensation or proprioception. Reflex examination confirmed the resolution of spinal shock; he remained in neurogenic shock and on a
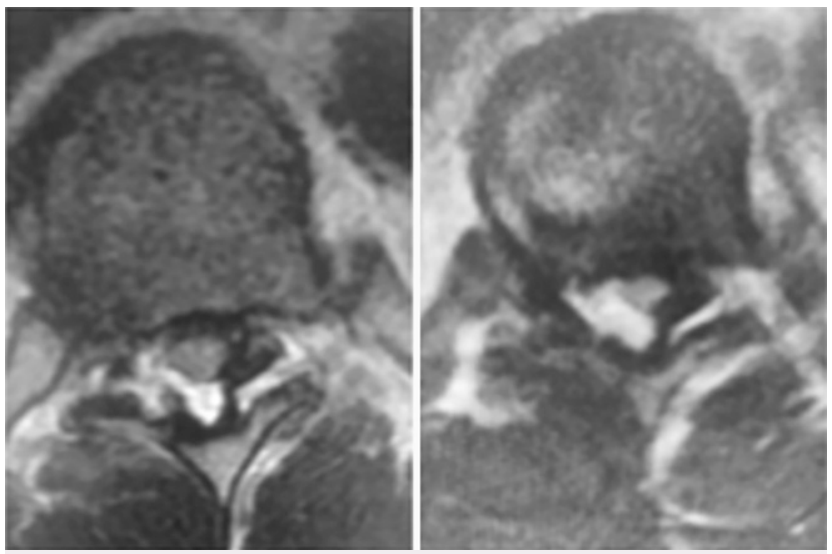

FIG. 2. Left: Axial T2 MRI immediately after injury. Right: After neurological deterioration on postinjury day 2 , a repeat MRI performed 48 hours after injury demonstrated interval development of epidural hematoma and thickening of the ligamentum flavum with new spinal cord compression and worsened spinal cord edema. norepinephrine infusion. Hypothermia was terminated after 24 hours because of extreme shivering.

On posttrauma day 2, the patient lost complete motor strength in the right lower extremity. He had pinprick sensory loss below T9T10 but slight proprioception and position sense in the right great toe. A repeat MRI revealed interval development of compression of the spinal cord at T9 at the apex of his scoliosis (Fig. 2 right) and worsening of T2 signal change at T8 with extensive spinal cord edema at T8-T10.

\section{Surgical Intervention}

Given the neurological deterioration and new imaging findings, he received spinal decompression and fixation. A T8-T10 posterior segmental instrumentation was performed with screws placed bilaterally at T8 and T10 and on the right at T9. After laminectomies of $\mathrm{T} 8$ and $\mathrm{T} 9$ and the superior aspect of T10, a left lateral extracavitary, transpedicular approach was performed at T9 to decompress the spinal cord at this level. The spinal cord was eccentric to the left in the spinal canal as a result of the scoliosis; the ligamentum flavum was thickened by hematoma, causing compression of the underlying spinal cord against the left T9 pedicle. The left T9 pedicle was resected, and full decompression of the spinal cord was achieved. A small epidural hematoma lateral and ventral to the spinal cord was also evacuated. Next, screws and rods were inserted to complete the bilateral T8 to T10 instrumentation. Local bone autograft and demineralized bone matrix completed the arthrodesis. At anesthesia induction, baseline upper extremity somatosensory evoked potentials (SSEPs) and motor evoked potentials (MEPs) were normal and lower extremity MEPs were absent. Baseline lower extremity SSEPs were present; the left leg response was relatively normal in latency and amplitude, and the right leg response, although present, was delayed in latency and smaller in amplitude. Lower extremity MEPs did not improve after the decompression.

The postoperative examination demonstrated no motor activity in either lower extremity. Again, there was vague proprioceptive and pressure sense on the right foot but absent pinprick sensation below T8. There was no change on examination on posttrauma day 3. Fortyeight hours after surgery (posttrauma day 4), ISNCSCI examination revealed T8 AIS grade C with intact rectal sensation and voluntary anal contraction. However, motor function was absent in both lower extremities, with some return of pinprick sensation bilaterally.

TABLE 1. Adjunctive therapeutic interventions

\begin{tabular}{|c|c|}
\hline Treatment & Treatment Regimen/Dose \\
\hline Hypothermia & $\begin{array}{c}35^{\circ} \mathrm{C} \text { core body temperature instituted with a } \\
\text { surface-cooling device }\end{array}$ \\
\hline Steroids & $\begin{array}{l}\text { Bracken methylprednisolone protocol of } 30 \mathrm{mg} / \mathrm{kg} \\
\text { for } 15 \mathrm{~min} \text { by infusion, } 45 \mathrm{~min} \text { pause, \& then } \\
23-\mathrm{hr} \text { maintenance infusion by } 5.4 \mathrm{mg} / \mathrm{kg}\end{array}$ \\
\hline HBOT & $\begin{array}{l}\text { One to two dives per day, for up to } 7 \text { days/wk; } \\
\text { each dive was at } 2.4 \text { ATA for } 90 \mathrm{~min}\end{array}$ \\
\hline $\begin{array}{l}\text { Omega-3 fatty } \\
\text { acids }\end{array}$ & $16 \mathrm{~g} /$ day $\&$ then maintenance dose of $2 \mathrm{~g} /$ day \\
\hline
\end{tabular}




\section{Adjunctive Medical Therapy}

Hyperbaric oxygen therapy (HBOT) was initiated on postoperative day 2, with one to two dives per day, up to 7 days a week, for a total of 30 dives over a period of 27 days. Each dive was at 2.4 ATA (atmospheres absolute) for 90 minutes. Initial serum omega-3 fatty acid level was $3.5 \%$, less than the desired level of $8 \%$ to $12 \%$. Nutritional supplementation was instituted with Omega Liquid Algae Concentrate (300 mg docosahexaenoic acid [DHA] and $150 \mathrm{mg}$ eicosapentaenoic acid [EPA] per 1,000 mg of Life's Omega 60 oil) for a total of 16 grams of EPA/DHA daily. Within 2 weeks of initiating omega-3 fatty acid supplementation, serum level reached $8.5 \%$. Table 1 summarizes the adjunct therapeutic options used.

\section{Rehabilitation Therapy}

Rehabilitation was initiated in the acute hospital setting starting postoperative day 6. On posttrauma day 14 (postoperative day 12), he was transferred to inpatient rehabilitation (IPR), where he completed various rehabilitation interventions. On admission to IPR, ISNCSCI examination revealed T9 AIS C incomplete paraplegia; by discharge, examination improved to T9 AIS D. Functional recovery, as measured by the functional independence measure, increased from 62 on admission to 114 on discharge. At discharge, he reached a modified independent level for all activities of daily living, transfers, and ultra-lightweight manual wheelchair mobility. He initially required bilateral knee-ankle-foot orthoses for ambulation, but by discharge he was able to ambulate up to 150 feet using left ankle-foot orthosis at a modified independent level using a wheeled
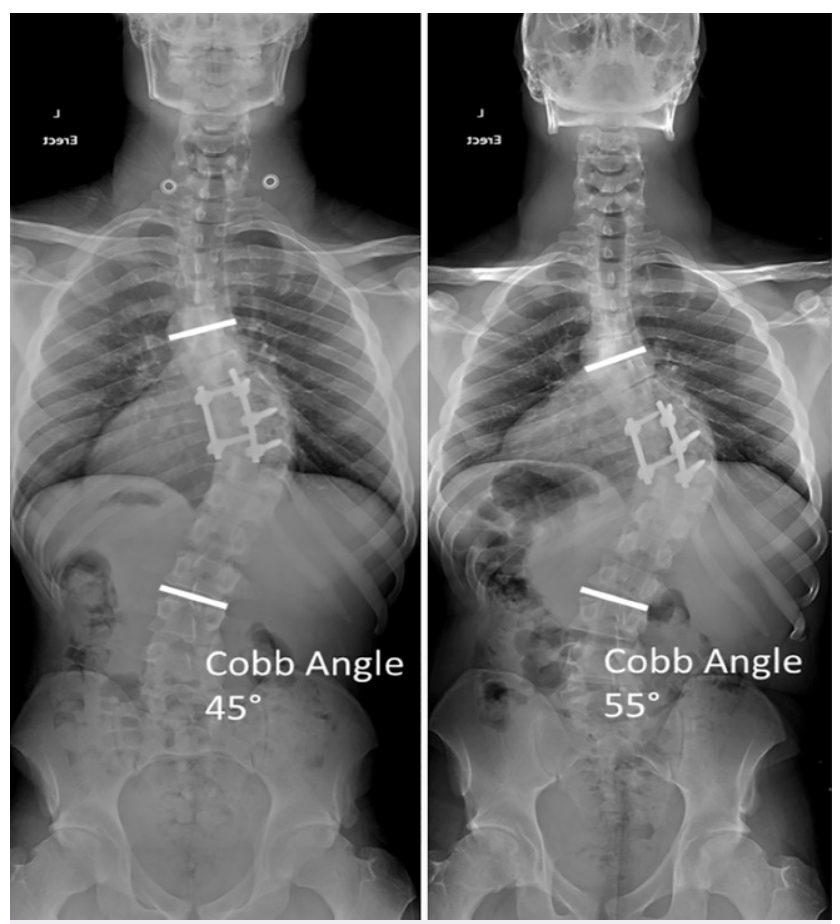

FIG. 3. Left: Standing posteroanterior long-cassette spine radiograph at 3 months after injury demonstrating the construct at T8-T10 and $45^{\circ}$ idiopathic thoracic dextroscoliosis. Right: Standing posteroanterior long-cassette spine radiograph at 6 months after injury revealed that the thoracic $\mathrm{Cobb}$ angle increased to $55^{\circ}$, concerning for interval development of neuromuscular scoliosis. Focused rehabilitation on core and paraspinal musculature was associated with no further change in patient's curve in the ensuing 2 years. walker. After IPR, he completed a total of 177 outpatient physical therapy (PT) visits, which were initiated with neuro-based PT, transitioned to a combination of neuro- and sports-based PT, and finally transitioned completely to a sports-based PT program.

At 4-month follow-up, standing long-cassette radiographs demonstrated a stable construct at T8-T9 (Fig. 3 left). Over the course of the first year after injury, he was transitioned from a left anklefoot orthosis to the use of an external electrical stimulation system for dorsiflexion, which was later discontinued. His 1-year ISNCSCI examination revealed normal lower extremity motor scores and mild sensory impairment, graded at T10 AIS D. At 18 months after injury, the patient had achieved significant motor and sensory recovery and was able to run and jump despite mild upper motor neuron spasticity and continued left knee recurvatum.

\section{Neuromuscular Scoliosis}

At 6 months after injury, a long-cassette scoliosis radiograph demonstrated progression of his thoracic curve: his thoracic Cobb angle was $55^{\circ}$, which was increased from $45^{\circ}$ degrees 3 months prior (Fig. 3 right). This was indicative of neuromuscular scoliosis, with curve progression in the setting of SCl. A modified Schrothmethod rehabilitation plan to strengthen his core and paraspinal musculature was instituted. At 12 and 24 months after injury, longcassette scoliosis radiographs confirmed no further progression of his idiopathic thoracic dextroscoliosis.

\section{Discussion}

\section{Observations}

A professional American football player sustained a T8 AIS A thoracic $\mathrm{SCl}$ in the setting of idiopathic scoliosis. Prompt intensive intervention, including surgery for a secondary neurological deterioration and prolonged intensive rehabilitation, facilitated an optimal functional recovery. Immediate surgery was not performed because there was no active neural element compression, and the spinal column fracture pattern was not considered unstable. Although surgical intervention was deferred at presentation, medical treatment was instituted immediately. The patient subsequently improved in neurological function. The secondary deterioration on posttrauma day 2 prompted a reevaluation, with identification of interval development of neural element compression. There was no hematoma on the first MRI; with the presence on the second MRI of an epidural hematoma as well as worsening of neurological conditions, surgery was urgently pursued. If there were no such deterioration, surgery would have been performed later and only if signs of instability emerged. The nature of the surgery (transpedicular decompression) produced iatrogenic instability; thus, spinal instrumentation was inserted during surgery.

\section{Idiopathic Scoliosis}

Idiopathic scoliosis occurs in $2 \%$ to $3 \%$ of the general population. ${ }^{7}$ Onset is typically in preadolescence. A nationwide study from Taiwan noted that an underlying spinal curvature disorder is associated with 2.2 times higher risk of a spinal injury from all causes of trauma. ${ }^{8} \mathrm{SCls}$ result when the spinal column fails under supraphysiologic loads. ${ }^{9}$ Classically, in axial loading injuries from forces to the crown of the head, the cervical spine must halt the momentum of the torso; the resultant spinal column failure and $\mathrm{SCl}$ are in the cervical region. ${ }^{10}$ An axial loading force to the crown of the head will exit the spine at its weakest point. In scoliosis, the lateral 


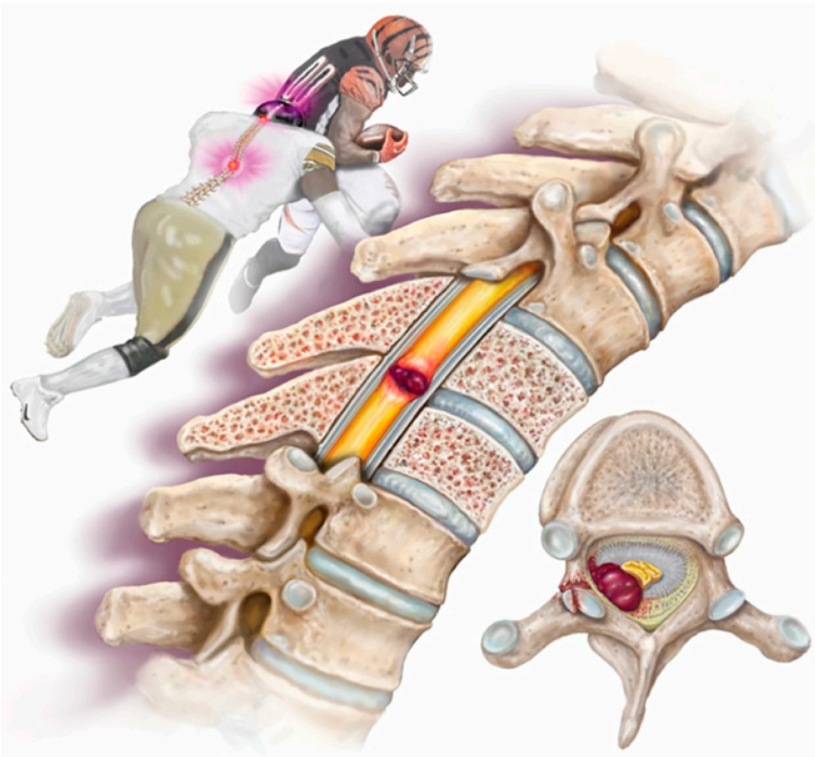

FIG. 4. Medical illustration depicting the mechanism of injury. An axial loading force vector at the crown of the helmet during a head-first tackle is transmitted through the spinal column, exiting at the apex of the thoracic scoliosis. The concavity of the apex at T9 was the site of load failure. Also in scoliosis, the spinal cord lies eccentric in the canal toward the concavity. Over the first 48 hours after, an epidural hematoma developed, causing spinal cord compression and worsening neurological insult, which led to surgical intervention.

eccentricity at the apex of the curve creates an alternative point of injury susceptibility. ${ }^{11}$ In the current case, the apex of the scoliotic curve at T9 was the site of load failure from axial forces to the crown of the helmet in a head-first tackle (Fig. 4). Although speculative, we question whether the scoliosis may have precipitated paraplegia but protected against quadriplegia, because in the absence of scoliosis, the point of biomechanical failure would otherwise be expected to have been in the cervical region.

\section{Hypothermia}

Hypothermia as a neuroprotective therapy for SCl is hypothesized to reduce the metabolic rate and prevent or ameliorate the inflammatory changes and other secondary injury mechanisms. ${ }^{12,13}$ Also, hypothermia reduces neutrophilic infiltration, inhibits microglia and the expression of tumor necrosis factor, and preserve synapses. ${ }^{14-16}$ Dididze et al. and Levi and colleagues at the University of Miami have reported on the relationship between hypothermia after $\mathrm{SCl}$ and increased likelihood of conversion to a higher AIS functional grade. $^{6,17}$ The use of systemic hypothermia has been reported in one other case of $\mathrm{SCl}$ in professional American football. ${ }^{18}$

\section{Steroids}

The National Acute Spinal Cord Injury Study Group (NASCIS) trials reported a significant neurological functional recovery in the patients receiving high-dose methylprednisolone given within 8 hours of injury. ${ }^{5,19}$ A meta-analysis of 4 randomized-controlled trials and 17 observational studies showed no significant short- or long-term beneficial effect on motor function, with some observational studies demonstrating a higher risk of gastrointestinal bleeding. ${ }^{20}$ Concerns are cited about the routine use of methylprednisolone in $\mathrm{SCl}$ because of a higher risk of complications, including infections, respiratory compromise, and hemorrhage. ${ }^{21,22}$ In this case, the patient was a young ( 25 years old), healthy, world-class athlete. We concluded that the potential benefits of steroid administration outweighed any potential risks.

\section{HBOT}

Ischemia is a significant secondary injury mechanism after SCl. Increasing oxygen delivery to the damaged spinal cord may help improve recovery. Asamoto et al. reported that 13 patients who received 85 minutes of HBOT at 2.0 ATA once daily for 10 days were found to have higher improvement rates indicated by the Neurological Cervical Spine Scale versus the non-HBOT group of 21 patients, ${ }^{23}$ with minimal to no adverse effects. HBOT promotes angiogenesis, enhances stem cell proliferation, is a potent antiinflammatory, and produces minimal risk in patients with acute $\mathrm{SCl}$. We used HBOT for 27 days starting from postoperative day 2 .

\section{Omega-3 Fatty Acids}

Animal models have demonstrated significant antiinflammatory benefits of omega-3 fatty acids by reducing cytokine production, apoptosis, axonal disruption, and free-radical damage. ${ }^{24-27}$ Given their safety profile, omega- 3 fatty acids may be considered an adjunct with the hope of reducing secondary damage due to inflammation, edema, and cytokines. In the current case, oral omega-3 fatty acid supplementation was instituted with serial measurement of serum omega-3 fatty acid level.

\section{Rehabilitation Intensity}

From 2015 to 2019, the average length of stay in IPR at the Spinal Cord Injury Model Systems Centers for someone with incomplete paraplegia was 32 days, demonstrating that this patient's duration of IPR was longer than average. ${ }^{28}$ Total PT hours completed during IPR were more than what has previously been reported in the literature for persons with paraplegia, ${ }^{29}$ although the types of rehabilitation activities were consistent with the standard of care. Outpatient therapy, up to 5 days a week over more than 2 years, was also of prolonged duration.

Intense activities-based rehabilitation therapies focusing on locomotor training are believed to promote plasticity via repetition to promote neural retraining for the specific task, in this case, ambulation. Sensory stimulation as a component of therapy, in combination with electrical stimulation and repetitive locomotor training, has been shown to improve walking after motor incomplete $\mathrm{SCl}{ }^{30}$ As outpatient therapies transitioned from neuro- to sports-based activities during the treatment program, the patient's intensity of aerobic performance increased over time and progressed from aided gait training to running. Previously it has been reported that high-intensity locomotor training may improve walking outcomes in persons with chronic motor incomplete $\mathrm{SCl}$ unrelated to the number of training sessions, indicating that the aerobic intensity of therapy sessions may additionally aid in outcomes. ${ }^{31}$ The transition of types of training therapies over time in parallel to motor recovery and associated improvement in ambulatory function suggests that the transition from neuro- to sports-based PT was appropriate. ${ }^{32}$

\section{Lessons}

To our knowledge, this is the first case of an NFL player sustaining a thoracic SCl while playing football. In addition, this is the first 
case of a professional football player with idiopathic scoliosis sustaining a spinal column injury and $\mathrm{SCl}$ at the apex of his structural curve. Because it represents a single case report, we do not purport, in any way, that any of the therapeutic interventions were singly therapeutic in his excellent recovery. We in no way imply that these treatments are standard of care for $\mathrm{SCl}$. We emphasize the point that scoliosis associated with $\mathrm{SCl}$ is a rare phenomenon and virtually unheard of in sports-related injury. This case report, in our opinion, is a critical piece for raising awareness and stimulating research into this concept and further researching the multiple therapeutic options available to help improve the outcomes of this typically devastating injury.

A case of catastrophic thoracic $\mathrm{SCl}$ in a professional American football player with idiopathic scoliosis was presented. Targeted medical and surgical intervention with intensive rehabilitation formed the treatment protocol with a favorable outcome. For $\mathrm{SCl}$ in healthy athletes, a combination of steroids, hypothermia, hyperbaric oxygen, nutritional supplementation, and intensive rehabilitation may be considered, with awareness that efficacy of each is speculative and requires further study.

\section{Acknowledgments}

We would like to acknowledge Christopher Venus, PT, and Joseph Everhart, PT, DT, for their contributions to the clinical care of this patient and for the rehabilitation data contained within the report. We would also like to thank Randal McKenzie for his work on the mechanism of injury illustrative figure.

\section{References}

1. National Spinal Cord Injury Statistical Center. Recent Trends in Cause of Spinal Cord Injury. University of Alabama at Birmingham; 2020.

2. Mall NA, Buchowski J, Zebala L, Brophy RH, Wright RW, Matava MJ. Spine and axial skeleton injuries in the National Football League. Am J Sports Med. 2012;40(8):1755-1761.

3. Hutton MJ, McGuire RA, Dunn R, et al. Catastrophic cervical spine injuries in contact sports. Global Spine J. 2016;6(7):721-734.

4. 2018 Football catastrophic report. Accessed December 10, 2019. https://nccsir.unc.edu/files/2019/10/Annual-FootballCatastrophic-2018-FINAL.pdf.

5. Bracken MB, Collins WF, Freeman DF, et al. Efficacy of methylprednisolone in acute spinal cord injury. JAMA. 1984;251(1):45-52.

6. Dididze M, Green BA, Dietrich WD, Vanni S, Wang MY, Levi AD. Systemic hypothermia in acute cervical spinal cord injury: a casecontrolled study. Spinal Cord. 2013;51(5):395-400.

7. Lonstein JE, Carlson JM. The prediction of curve progression in untreated idiopathic scoliosis during growth. J Bone Joint Surg Am. 1984;66(7):1061-1071.

8. Kuo YL, Chung $\mathrm{CH}$, Huang TW, et al. Association between spinal curvature disorders and injury: a nationwide population-based retrospective cohort study. BMJ Open. 2019;9(1):e023604.

9. White AA, Panjabi MM. Clinical Biomechanics of the Spine. J.B. Lippincott; 1990.

10. Miura T, Panjabi MM, Cripton PA. A method to simulate in vivo cervical spine kinematics using in vitro compressive preload. Spine (Phila Pa 1976). 2002;27(1):43-48.

11. Whyte $T$, Melnyk $A D$, Van Toen $C$, et al. A neck compression injury criterion incorporating lateral eccentricity. Sci Rep. 2020;10(1):7114.

12. Ha KY, Kim YH. Neuroprotective effect of moderate epidural hypothermia after spinal cord injury in rats. Spine (Phila Pa 1976). 2008;33(19):2059-2065.
13. Seo JY, Kim YH, Kim JW, Kim SI, Ha KY. Effects of therapeutic hypothermia on apoptosis and autophagy after spinal cord injury in rats. Spine (Phila Pa 1976). 2015;40(12):883-890.

14. Bandschapp O, laizzo PA. Induction of therapeutic hypothermia requires modulation of thermoregulatory defenses. Ther Hypothermia Temp Manag. 2011;1(2):77-85.

15. Chatzipanteli K, Yanagawa Y, Marcillo AE, Kraydieh S, Yezierski RP, Dietrich WD. Posttraumatic hypothermia reduces polymorphonuclear leukocyte accumulation following spinal cord injury in rats. J Neurotrauma. 2000;17(4):321-332.

16. Lo TP Jr, Cho KS, Garg MS, et al. Systemic hypothermia improves histological and functional outcome after cervical spinal cord contusion in rats. J Comp Neurol. 2009;514(5):433-448.

17. Levi AD, Casella G, Green BA, et al. Clinical outcomes using modest intravascular hypothermia after acute cervical spinal cord injury. Neurosurgery. 2010;66(4):670-677.

18. Cappuccino A, Bisson LJ, Carpenter B, Snyder K, Cappuccino H. Systemic hypothermia as treatment for an acute cervical spinal cord injury in a professional football player: 9-year follow-up. Am J Orthop. 2017;46(2):E79-E82.

19. Bracken MB, Shepard MJ, Collins WF, et al. A randomized, controlled trial of methylprednisolone or naloxone in the treatment of acute spinal-cord injury. Results of the Second National Acute Spinal Cord Injury Study. N Engl J Med. 1990;322(20):1405-1411.

20. Evaniew N, Belley-Côté EP, Fallah N, Noonan VK, Rivers CS, Dvorak MF. Methylprednisolone for the treatment of patients with acute spinal cord injuries: a systematic review and meta-analysis. J Neurotrauma. 2016;33(5):468-481.

21. Hurlbert RJ, Hadley MN, Walters BC, et al. Pharmacological therapy for acute spinal cord injury. Neurosurgery. 2015;76(suppl 1): S71-S83.

22. Ito $Y$, Sugimoto $Y$, Tomioka M, Kai N, Tanaka M. Does high dose methylprednisolone sodium succinate really improve neurological status in patient with acute cervical cord injury? A prospective study about neurological recovery and early complications. Spine (Phila Pa 1976). 2009;34(20):2121-2124.

23. Asamoto $S$, Sugiyama H, Doi $H$, lida M, Nagao T, Matsumoto $K$. Hyperbaric oxygen (HBO) therapy for acute traumatic cervical spinal cord injury. Spinal Cord. 2000;38(9):538-540.

24. King VR, Huang WL, Dyall SC, Curran OE, Priestley JV, MichaelTitus AT. Omega- 3 fatty acids improve recovery, whereas omega- 6 fatty acids worsen outcome, after spinal cord injury in the adult rat J Neurosci. 2006;26(17):4672-4680.

25. Bi J, Chen C, Sun P, Tan H, Feng F, Shen J. Neuroprotective effect of omega-3 fatty acids on spinal cord injury induced rats. Brain Behav. 2019;9(8):e01339.

26. Oliveira KM, Lavor MS, Silva CM, et al. Omega-conotoxin MVIIC attenuates neuronal apoptosis in vitro and improves significant recovery after spinal cord injury in vivo in rats. Int J Clin Exp Pathol. 2014;7(7):3524-3536.

27. Mills JD, Bailes JE, Sedney CL, Hutchins H, Sears B. Omega-3 fatty acid supplementation and reduction of traumatic axonal injury in a rodent head injury model. $J$ Neurosurg. 2011;114(1):77-84.

28. National Spinal Cord Injury Statistical Center. Annual statistical report for the spinal cord injury model systems. Accessed December 10, 2019. https://www.nscisc.uab.edu

29. Taylor-Schroeder S, LaBarbera J, McDowell S, et al. The SCIRehab project: treatment time spent in $\mathrm{SCl}$ rehabilitation. Physical therapy treatment time during inpatient spinal cord injury rehabilitation. J Spinal Cord Med. 2011;34(2):149-161.

30. Behrman AL, Ardolino EM, Harkema SJ. Activity-based therapy: from basic science to clinical application for recovery after spinal cord injury. J Neurol Phys Ther. 2017;41(suppl 3):S39-S45.

31. Brazg G, Fahey M, Holleran CL, et al. Effects of training intensity on locomotor performance in individuals with chronic spinal cord 
injury: a randomized crossover study. Neurorehabil Neural Repair. 2017;31(10-11):944-954.

32. Franz M, Richner L, Wirz M, et al. Physical therapy is targeted and adjusted over time for the rehabilitation of locomotor function in acute spinal cord injury interventions in physical and sports therapy. Spinal Cord. 2018;56(2):158-167.

\section{Disclosures}

Dr. Maroon is a Consultant, National Football League; Head, Neck and Spine Committee Team neurosurgeon; The Pittsburgh Steelers Football Club Medical Director; WWE. Dr. N. Agarwal reports other from Thieme Medical Publishers outside the submitted work. Dr. Okonkwo is Consulting Neurosurgeon, The Pittsburgh Steelers Football Club. This research was supported in part by donations to the Neuroscience Research Foundation from Lewis Topper, Dennis and Rose Heindl, Nelson Peltz, John Garcia, and the Tampa Bay Buccaneers Football Club.

\section{Author Contributions}

Conception and design: Maroon, N. Agarwal, Okonkwo. Acquisition of data: Maroon, Faramand, Harrington, N. Agarwal, Norwig, Okonkwo. Analysis and interpretation of data: N. Agarwal, Harrington, Okonkwo. Drafting the article: Maroon, Faramand, N. Agarwal, Harrington,

Okonkwo. Critically revising the article: Maroon, N. Agarwal, Harrington, V. Agarwal, Okonkwo. Reviewed submitted version of manuscript: all authors. Approved the final version of the manuscript on behalf of all authors: Maroon. Statistical analysis: Okonkwo. Administrative/technical/ material support: Maroon, Okonkwo. Study supervision: Maroon, Okonkwo.

\section{Correspondence}

Joseph C. Maroon: University of Pittsburgh Medical Center, Pittsburgh, PA. maroonjc@gmail.com. 